\title{
Extensions of Dupire Formula: Stochastic Interest Rates and Stochastic Local Volatility
}

\author{
Orcan Ögetbil, and Bernhard Hientzsch ${ }^{\dagger}$ \\ Corporate Model Risk, Wells Fargo Bank
}

\begin{abstract}
We derive generalizations of Dupire formula to the cases of general stochastic drift and/or stochastic local volatility. First, we handle a case in which the drift is given as difference of two stochastic short rates. Such a setting is natural in foreign exchange context where the short rates correspond to the short rates of the two currencies, equity single-currency context with stochastic dividend yield, or commodity context with stochastic convenience yield. We present the formula both in a call surface formulation as well as total implied variance formulation where the latter avoids calendar spread arbitrage by construction. We provide derivations for the case where both short rates are given as single factor processes and present the limits for a single stochastic rate or all deterministic short rates. The limits agree with published results. Then we derive a formulation that allows a more general stochastic drift and diffusion including one or more stochastic local volatility terms. In the general setting, our derivation allows the computation and calibration of the leverage function for stochastic local volatility models. Despite being implicit, the generalized Dupire formulae can be used numerically in a fixed-point iterative scheme.
\end{abstract}

Keywords Dupire Equation, Local Volatility, Stochastic Rates, Stochastic Local Volatility

AMS 91G20, 91G30

\section{Introduction}

Risk neutral pricing frameworks aim to establish methodologies for producing prices consistent with market data available as of valuation time. As a standard approach, practitioners consider parametric models to map market quotes to time and space dependent model parameters. The single parameter Black-Scholes model, for instance, gives European vanilla option prices as a function of implied volatility. In a sense, having an implied volatility surface spanning a range of strikes and maturities is equivalent to knowing the prices of

*orcan.ogetbil@wellsfargo.com

†bernhard.hientzsch@wellsfargo.com 
European vanilla options, whose payoffs depend on the value of the underlier solely at maturity, for the same strikes and maturities. This in turn amounts to knowing the risk-neutral probability density of the underlier at given future times conditioned on its present value. In this paradigm, the bulk of the work in developing a methodology to price European vanilla option instruments written on the same underlier lies in the construction of the implied volatility surface.

The benefits of formulating the risk-neutral probability density as a function of time and underlying spot value, however, go beyond the ability to price European vanilla options. To price more complex options, whose payoffs depend not only on the terminal value of the underlier, but also on its intermediate values, one can make use of the risk neutral densities implied by the market prices of European vanilla options at the intermediate times. Dupire [1], and Derman and Kani [2] showed that there is a unique diffusion process that implies risk neutral probabilities consistent with the European vanilla option market quotes. Dupire's formula provides a map in a non-parametric way between European vanilla option market prices and the diffusion coefficient under the assumption of deterministic interest rates.

Various authors considered extensions of the local volatility formulation, or embedding local volatility into more complex hybrid models. These efforts include incorporating jumpdiffusion processes [3], representing the effect of one stochastic interest rate as a bias to the fully deterministic rate model [4, local volatility with single interest rate following a Vasicek model [5, introducing stochasticity to local volatility [6, 7, 8, 9], and embedding local volatility into a nonlinear McKean SDE [10]. We refer to these papers for historical background on the development of local volatility based models.

The primary goal of this paper is to provide a self-contained, detailed derivation for the case of a drift given as the difference of two short rates driven by single factor processes, and to provide a new generalization to a case with very general stochastic drift and diffusion terms. In Section 2, we construct the direct extension of the standard local volatility model to cover stochastic domestic and foreign interest rates in a foreign exchange (FX) derivatives setting. The result is presented in both vanilla call option price (2.18) and total BlackScholes implied variance (A.10) formulations. We also consider the limiting cases, with one or both rates being deterministic, to recover results that can be found in literature ((2.20), (2.21), (A.11), and (A.12), respectively). Section 3 further extends the model to allow drift and diffusion functions of general form with arbitrary number of stochastic factors. The general setting is given by (3.1) and the corresponding Dupire formula in (3.6). This general setup has greater coverage than the examples found in literature as the interest rates are not assumed to follow particular processes, such as short rate models; the assumption we have is that the discount factor and the asset volatility are adapted functions of the Itô processes in the SDE system. An outline of the derivation for the vanilla call option formulation with Hull-White short rates is presented in [11. In contrast, we endeavor to present a complete and thorough derivation for the general case. The rest of the section discusses specific examples and implications for the leverage function in the stochastic local volatility models as well as connections to Gyöngy's lemma. We chose to present self-contained details of the derivations in the flow of the derivations since that gives more insight into the actual foundation and possible extensions. In Section 4, we demonstrate a calibration scheme with fixed-point iteration for the local volatility model subject to stochastic rates, following Linear Gaussian Model (LGM) processes; and study the convergence of the iterations, and 
accuracy of the pricing with the calibrated local volatility surface.

\section{Local Volatility Model with Two Stochastic Interest Rates}

\subsection{Model Setup}

We assume the existence of a domestic risk neutral measure $\mathbb{Q}^{\text {DRN }}$ that has the money market account $B_{t}^{d}$ as the numéraire, which accrues at some computable short rate $r_{t}^{d}$ as $d B_{t}^{d}=r_{t}^{d} B_{t}^{d} d t$. Dupire introduces a state dependent diffusion coefficient $\sigma^{\mathrm{LV}}\left(S_{t}, t\right)$ that uniquely describes the distribution of the state variable $S_{t}$ for each time $t$, conditioned on the initial value $S_{0}$. Accordingly, there is a risk-neutral spot process that is compatible with observed market skew and allows a complete model. This is commonly referred to as local volatility process [1], driven by the Brownian motion $W_{t}^{S(\mathrm{DRN})}$ under the domestic risk neutral measure $\mathbb{Q}^{\mathrm{DRN}}$,

$$
d S_{t}=\mu_{t} S_{t} d t+\sigma^{\mathrm{LV}}\left(S_{t}, t\right) S_{t} d W_{t}^{S(\mathrm{DRN})} .
$$

Dupire's formula gives the function $\sigma^{\mathrm{LV}}(\cdot, \cdot)$ in terms of call and/or put option prices, or equivalently, implied volatility or total implied variance.

The original work of Dupire [1] assumes zero interest rates $\left(\mu_{t}=0\right)$, while the independent study of Derman and Kani [2] introduces deterministic interest rates $\left(\mu_{t}=\mu(t)\right)$. In the latter setup, the drift term $\mu_{t}$ is assumed to be the instantaneous forward rate of maturity $t$ implied from the yield curve, which is a deterministic function of time. In this paper we relax this constraint and let this term be stochastic. In particular, we are interested in a model with two stochastic terms that comprise a drift of the form $\mu_{t}=\mu_{t}^{1}-\mu_{t}^{2}$. One can consider the pair $\mu_{t}^{1}, \mu_{t}^{2}$ as interest rate/dividend rate in equities setup, or domestic rate/foreign rate in foreign exchange setup. In this section, without loss of generality, we will use the conventions of the latter. In particular, $\mu_{t}^{1}=r_{t}^{d}$ and $\mu_{t}^{2}=r_{t}^{f}$ denote the domestic and foreign short rates, respectively. Under the domestic risk neutral measure $\mathbb{Q}^{\mathrm{DRN}}$, these rates follow single factor processes of the generic form

$$
\begin{aligned}
& d r_{t}^{d}=\alpha^{d}(\omega, t) d t+\sigma^{d}(\omega, t) d W_{t}^{d(\mathrm{DRN})}, \\
& d r_{t}^{f}=\alpha^{f}(\omega, t) d t+\sigma^{f}(\omega, t) d W_{t}^{f(\mathrm{DRN})},
\end{aligned}
$$

where $\alpha^{d}, \sigma^{d}, \alpha^{f}$, and $\sigma^{f}$ are bounded functions of a general set of stochastic factors $\omega$. Our model admits three Brownian motions and we set the three pairs of correlations 1 as $d\left\langle W^{S}, W^{d}\right\rangle_{t}=\rho^{S d} d t, \quad d\left\langle W^{S}, W^{f}\right\rangle_{t}=\rho^{S f} d t$, and $\quad d\left\langle W^{d}, W^{f}\right\rangle_{t}=\rho^{d f} d t$. Note that the short rate stochastic differential equations (SDEs) are typically written in the risk neutral measure of their own currency. Here, drift adjustment in the foreign short rate process due to the change from foreign risk neutral measure to domestic risk neutral measure is absorbed into the term $\alpha^{f}$. When required by our computations below, we assume that the functions $\sigma^{\mathrm{LV}}, \alpha^{d}, \sigma^{d}, \alpha^{f}$, and $\sigma^{f}$ are twice differentiable with respect to the arguments over their entire ranges.

\footnotetext{
${ }^{1}$ In general, the correlations can be time-dependent; or they can even be generalized to stochastic processes as we shall see in Section 3 The nature of the correlations does not have any impact on our result, thus we keep their notation simple.
} 


\subsection{Fokker-Planck equation}

Let $V_{T} \equiv V\left(S_{T}, r_{T}^{d}, r_{T}^{f}\right)$ be a twice differentiable $\mathbb{R}^{3} \rightarrow \mathbb{R}$ test function. For each given $T$, the discounted value of a European option maturing at $T$ with the payoff equal to that of the test function $V_{T}$ is a martingale under the domestic risk neutral measure $\mathbb{Q}^{\mathrm{DRN}}$,

$$
\frac{V_{0}}{B_{0}^{d}}=V_{0}=\mathbf{E}^{\mathbb{Q}^{\mathrm{DRN}}}\left[\frac{V_{T}}{B_{T}^{d}}\right]
$$

where $B_{T}^{d}=\exp \left[\int_{0}^{T} r_{u}^{d} d u\right]$ is the domestic money market account, by means of which we

can define $D_{T} \equiv \frac{1}{B_{T}^{d}}$ as the corresponding discount factor. In the (domestic) $T$-forward measure $\mathbb{Q}^{\mathrm{T}}$, the zero coupon bond $P^{d}(0, T)$ maturing at time $T$ is taken as the numéraire

$$
P^{d}(t, T)=\mathbf{E}^{\mathbb{Q}^{\mathrm{DRN}}}\left[\frac{D_{T}}{D_{t}} \mid \mathcal{F}_{t}\right], \text { and } \frac{d \mathbb{Q}^{\mathrm{T}}}{d \mathbb{Q}^{\mathrm{DRN}}}=\frac{D_{T}}{\mathbf{E}^{\mathbb{Q}^{\mathrm{DRN}}}\left[D_{T}\right]}=\frac{D_{T}}{P^{d}(0, T)}
$$

where the filtration $\left\{\mathcal{F}_{t}, t \geq 0\right\}$ governs information arrival. Thus

$$
\begin{aligned}
\mathbf{E}^{\mathbb{Q}^{\mathrm{DRN}}}\left[D_{T} V_{T}\right] & =P^{d}(0, T) \mathbf{E}^{\mathbb{Q}^{T}}\left[V_{T}\right] \\
& =P^{d}(0, T) \iiint V\left(S_{T}, r_{T}^{d}, r_{T}^{f}\right) \Phi^{T}\left(S_{T}, r_{T}^{d}, r_{T}^{f}, T\right) d S_{T} d r_{T}^{d} d r_{T}^{f},
\end{aligned}
$$

where $\Phi^{T}\left(S_{T}, r_{T}^{d}, r_{T}^{f}, T\right)$ denotes the $T$-forward measure probability density. We assume the probability density function to be sufficiently tractable; in particular, it is bounded; and it is differentiable with respect to time and twice differentiable with respect to its spatial arguments. Notationwise, here and in what is below, the integrals written without explicit limits are meant to be taken over the entire domain, which is $(-\infty, \infty)$ in most cases.

One can integrate the full $T$-forward probability density $\Phi^{T}$ over the entire ranges of $r_{T}^{d}$ and $r_{T}^{f}$ to get the marginal $T$-forward probability density $q^{T}$ of $S_{T}$ over time,

$$
q^{T}\left(S_{T}, T\right)=\iint \Phi^{T}\left(S_{T}, r_{T}^{d}, r_{T}^{f}, T\right) d r_{T}^{d} d r_{T}^{f}
$$

The marginal $T$-forward distribution has the time derivative

$$
\frac{\partial q^{T}\left(S_{T}, T\right)}{\partial T}=\iint \frac{\partial \Phi^{T}\left(S_{T}, r_{T}^{d}, r_{T}^{f}, T\right)}{\partial T} d r_{T}^{d} d r_{T}^{f}
$$

Next, we apply Itô's lemma to the discounted test function,

$$
\begin{aligned}
\frac{d\left(D_{T} V_{T}\right)}{D_{T}}= & {\left[-r_{T}^{d} V_{T}+\frac{1}{2}\left(\sigma_{T}^{\mathrm{LV}}\right)^{2} S_{T}^{2} \frac{\partial^{2} V_{T}}{\partial S_{T}^{2}}+\left(r_{T}^{d}-r_{T}^{f}\right) S_{T} \frac{\partial V_{T}}{\partial S_{T}}\right.} \\
& +\frac{1}{2}\left(\sigma_{T}^{d}\right)^{2} \frac{\partial^{2} V_{T}}{\partial\left(r_{u}^{d}\right)^{2}}+\alpha_{T}^{d} \frac{\partial V_{T}}{\partial r_{u}^{d}}+\frac{1}{2}\left(\sigma_{T}^{f}\right)^{2} \frac{\partial^{2} V_{T}}{\partial\left(r_{T}^{f}\right)^{2}}+\alpha_{T}^{f} \frac{\partial V_{T}}{\partial r_{T}^{f}} \\
& \left.+\rho^{S d} S_{T} \sigma_{T}^{\mathrm{LV}} \sigma_{T}^{d} \frac{\partial^{2} V_{T}}{\partial S_{T} \partial r_{T}^{d}}+\rho^{S f} S_{T} \sigma_{T}^{\mathrm{LV}} \sigma_{T}^{f} \frac{\partial^{2} V_{T}}{\partial S_{T} \partial r_{T}^{f}}+\rho^{d f} \sigma_{T}^{d} \sigma_{T}^{f} \frac{\partial^{2} V_{T}}{\partial r_{T}^{d} \partial r_{T}^{f}}\right] d T \\
& +\sigma_{T}^{\mathrm{LV}} S_{T} \frac{\partial V_{T}}{\partial S_{T}} d W_{T}^{S(\mathrm{DRN})}+\sigma_{T}^{d} \frac{\partial V_{T}}{\partial r_{T}^{d}} d W_{T}^{d(\mathrm{DRN})}+\sigma_{T}^{f} \frac{\partial V_{T}}{\partial r_{T}^{f}} d W_{T}^{f(\mathrm{DRN})} .
\end{aligned}
$$


Here and below we use the convention $\sigma_{T}^{\mathrm{LV}} \equiv \sigma^{\mathrm{LV}}\left(S_{T}, T\right), \alpha_{T}^{d} \equiv \alpha^{d}(\omega, T), \sigma_{T}^{d} \equiv \sigma^{d}(\omega, T)$, $\alpha_{T}^{f} \equiv \alpha^{f}(\omega, T)$, and $\sigma_{T}^{f} \equiv \sigma^{f}(\omega, T)$ for notational brevity. By taking the expectation in $\mathbb{Q}^{\mathrm{DRN}}$, we find

$$
\begin{aligned}
\frac{\partial \mathbf{E}^{\mathbb{Q}^{\mathrm{DRN}}}\left[D_{T} V_{T}\right]}{\partial T}=\mathbf{E}^{\mathbb{Q}^{\mathrm{DRN}}}\left[D_{T}(\right. & -r_{T}^{d} V_{T}+\frac{1}{2}\left(\sigma_{T}^{\mathrm{LV}}\right)^{2} S_{T}^{2} \frac{\partial^{2} V_{T}}{\partial S_{T}^{2}}+\left(r_{T}^{d}-r_{T}^{f}\right) S_{T} \frac{\partial V_{T}}{\partial S_{T}} \\
& +\frac{1}{2}\left(\sigma_{T}^{d}\right)^{2} \frac{\partial^{2} V_{T}}{\partial\left(r_{T}^{d}\right)^{2}}+\alpha_{T}^{d} \frac{\partial V_{T}}{\partial r_{T}^{d}}+\frac{1}{2}\left(\sigma_{T}^{f}\right)^{2} \frac{\partial^{2} V_{T}}{\partial\left(r_{T}^{f}\right)^{2}}+\alpha_{T}^{f} \frac{\partial V_{T}}{\partial r_{T}^{f}} \\
& +\rho^{S d} S_{T} \sigma_{T}^{\mathrm{LV}} \sigma_{T}^{d} \frac{\partial^{2} V_{T}}{\partial S_{T} \partial r_{T}^{d}}+\rho^{S f} S_{T} \sigma_{T}^{\mathrm{LV}} \sigma_{T}^{f} \frac{\partial^{2} V_{T}}{\partial S_{T} \partial r_{T}^{f}} \\
& \left.\left.+\rho^{d f} \sigma_{T}^{d} \sigma_{T}^{f} \frac{\partial^{2} V_{T}}{\partial r_{T}^{d} \partial r_{T}^{f}}\right)\right]
\end{aligned}
$$

On the other hand, we differentiate (2.5) with respect to $T$ to get

$$
\begin{aligned}
\frac{\partial\left(P^{d}(0, T) \mathbf{E}^{\mathbb{Q}^{T}}\left[V_{T}\right]\right)}{\partial T}= & \frac{\partial P^{d}(0, T)}{\partial T} \iiint V_{T} \Phi^{T} d S_{T} d r_{T}^{d} d r_{T}^{f} \\
& +P^{d}(0, T) \iiint V_{T} \frac{\partial \Phi^{T}}{\partial T} d S_{T} d r_{T}^{d} d r_{T}^{f},
\end{aligned}
$$

By combining (2.8) and (2.9) we arrive at

$$
\begin{gathered}
r 0=\frac{\partial P^{d}(0, T)}{\partial T} \iiint V_{T} \Phi^{T} d S_{T} d r_{T}^{d} d r_{T}^{f}+P^{d}(0, T) \iiint V_{T} \frac{\partial \Phi^{T}}{\partial T} d S_{T} d r_{T}^{d} d r_{T}^{f} \\
+P^{d}(0, T) \iiint \Phi^{T}\left[r_{T}^{d} V_{T}-\frac{1}{2}\left(\sigma_{T}^{\mathrm{LV}}\right)^{2} S_{T}^{2} \frac{\partial^{2} V_{T}}{\partial S_{T}^{2}}-\left(r_{T}^{d}-r_{T}^{f}\right) S_{T} \frac{\partial V_{T}}{\partial S_{T}}\right. \\
-\frac{1}{2}\left(\sigma_{T}^{d}\right)^{2} \frac{\partial^{2} V_{T}}{\partial\left(r_{T}^{d}\right)^{2}}-\alpha_{T}^{d} \frac{\partial V_{T}}{\partial r_{T}^{d}}-\frac{1}{2}\left(\sigma_{T}^{f}\right)^{2} \frac{\partial^{2} V_{T}}{\partial\left(r_{T}^{f}\right)^{2}}-\alpha_{T}^{f} \frac{\partial V_{T}}{\partial r_{T}^{f}} \\
-\rho^{S d} S_{T} \sigma_{T}^{\mathrm{LV}} \sigma_{T}^{d} \frac{\partial^{2} V_{T}}{\partial S_{T} \partial r_{T}^{d}}-\rho^{S f} S_{T} \sigma_{T}^{\mathrm{LV}} \sigma_{T}^{f} \frac{\partial^{2} V_{T}}{\partial S_{T} \partial r_{T}^{f}} \\
\left.-\rho^{d f} \sigma_{T}^{d} \sigma_{T}^{f} \frac{\partial^{2} V_{T}}{\partial r_{T}^{d} \partial r_{T}^{f}}\right] d S_{T} d r_{T}^{d} d r_{T}^{f} .
\end{gathered}
$$

Using the definition of the instantaneous forward rate

$$
f^{i}(0, T) \equiv-\frac{\partial \log P^{i}(0, T)}{\partial T}=-\frac{1}{P^{i}(0, T)} \frac{\partial P^{i}(0, T)}{\partial T}
$$


with $i=d, f$, we can reformulate this as

$$
\begin{aligned}
0=\iiint\left[V_{T} \frac{\partial \Phi^{T}}{\partial T}+\Phi^{T}\right. & \left\{\left(r_{T}^{d}-f^{d}(0, T)\right) V_{T}\right. \\
& -\frac{1}{2}\left(\sigma_{T}^{\mathrm{LV}}\right)^{2} S_{T}^{2} \frac{\partial^{2} V_{T}}{\partial S_{T}^{2}}-\left(r_{T}^{d}-r_{T}^{f}\right) S_{T} \frac{\partial V_{T}}{\partial S_{T}} \\
& -\frac{1}{2}\left(\sigma_{T}^{d}\right)^{2} \frac{\partial^{2} V_{T}}{\partial\left(r_{T}^{d}\right)^{2}}-\alpha_{T}^{d} \frac{\partial V_{T}}{\partial r_{T}^{d}}-\frac{1}{2}\left(\sigma_{T}^{f}\right)^{2} \frac{\partial^{2} V_{T}}{\partial\left(r_{T}^{f}\right)^{2}}-\alpha_{T}^{f} \frac{\partial V_{T}}{\partial r_{T}^{f}} \\
& -\rho^{S d} S_{T} \sigma_{T}^{\mathrm{LV}} \sigma_{T}^{d} \frac{\partial^{2} V_{T}}{\partial S_{T} \partial r_{T}^{d}}-\rho^{S f} S_{T} \sigma_{T}^{\mathrm{LV}} \sigma_{T}^{f} \frac{\partial^{2} V_{T}}{\partial S_{T} \partial r_{T}^{f}} \\
& \left.\left.-\rho^{d f} \sigma_{T}^{d} \sigma_{T}^{f} \frac{\partial^{2} V_{T}}{\partial r_{T}^{d} \partial r_{T}^{f}}\right\}\right] d S_{T} d r_{T}^{d} d r_{T}^{f} .
\end{aligned}
$$

The collection of zero coupon bond prices $P^{i}$ with maturities sequenced over a time grid is called a discount curve. The instantaneous forward rates $f^{i}$ can be evaluated along given discount curves which are used as standard input data in various pricing and other financial models.

We integrate by parts the terms that have the partial derivatives of $V_{T}$ appearing in (2.11). Noting that the boundary terms vanish as we assume $\Phi^{T}$ and its derivatives tend to zero fast enough as its arguments approach the integration limits, we can derive the following identities by integrating by parts

$$
\begin{gathered}
\int \Phi^{T} f(\cdot) \frac{\partial^{2} V_{T}}{\partial u^{2}} d u=\int \frac{\partial^{2}\left(\Phi^{T} f(\cdot)\right)}{\partial u^{2}} V_{T} d u, \\
\int \Phi^{T} f(\cdot) \frac{\partial V_{T}}{\partial u} d u=-\int \frac{\partial\left(\Phi^{T} f(\cdot)\right)}{\partial u} V_{T} d u, \\
\int \Phi^{T} f(\cdot) \frac{\partial^{2} V_{T}}{\partial u \partial v} d u d v=\int \frac{\partial^{2}\left(\Phi^{T} f(\cdot)\right)}{\partial u \partial v} V_{T} d u d v,
\end{gathered}
$$

for a sufficiently well behaved (bounded, continuous, differentiable) function $f$ of spatial coordinates $u$ and $v$, e.g. representing $S_{T}, r_{T}^{d}, r_{T}^{f}$ in our setup. Thus (2.11) can be written as

$$
\begin{aligned}
0=\iiint V_{T} & \left\{\frac{\partial \Phi^{T}}{\partial T}+\Phi^{T}\left(r_{T}^{d}-f^{d}(0, T)\right)-\frac{1}{2} \frac{\partial^{2}\left(\Phi^{T}\left(\sigma_{T}^{\mathrm{LV}}\right)^{2} S_{T}^{2}\right)}{\partial S_{T}^{2}}+\left(r_{T}^{d}-r_{T}^{f}\right) \frac{\partial\left(\Phi^{T} S_{T}\right)}{\partial S_{T}}\right. \\
& -\frac{1}{2} \frac{\partial^{2}\left(\Phi\left(\sigma_{T}^{d}\right)^{2}\right)}{\partial\left(r_{T}^{d}\right)^{2}}+\frac{\partial\left(\Phi^{T} \alpha_{T}^{d}\right)}{\partial r_{T}^{d}}-\frac{1}{2} \frac{\partial^{2}\left(\Phi^{T}\left(\sigma_{T}^{f}\right)^{2}\right)}{\partial\left(r_{T}^{f}\right)^{2}}+\frac{\partial\left(\Phi^{T} \alpha_{T}^{f}\right)}{\partial r_{T}^{f}} \\
& -\frac{\partial^{2}\left(\Phi^{T} \rho^{S d} S_{T} \sigma_{T}^{\mathrm{LV}} \sigma_{T}^{d}\right)}{\partial S_{T} \partial r_{T}^{d}}-\frac{\partial^{2}\left(\Phi^{T} \rho^{S f} S_{T} \sigma_{T}^{\mathrm{LV}} \sigma_{T}^{f}\right)}{\partial S_{T} \partial r_{T}^{f}} \\
& \left.-\frac{\partial^{2}\left(\Phi^{T} \rho^{d f} \sigma_{T}^{d} \sigma_{T}^{f}\right)}{\partial r_{T}^{d} \partial r_{T}^{f}}\right\} d S_{T} d r_{T}^{d} d r_{T}^{f} .
\end{aligned}
$$

Since the above equation holds for any $V_{T}$, the term inside the braces must vanish. This leads us to the Fokker-Planck (forward Kolmogorov) equation [12], which describes the 
evolution of the probability density function $\Phi^{T}\left(S_{T}, r_{T}^{d}, r_{T}^{f}, T\right)$ of the underlying factors over time,

$$
\begin{aligned}
0= & \frac{\partial \Phi^{T}}{\partial T}+\Phi^{T}\left(r_{T}^{d}-f^{d}(0, T)\right)-\frac{1}{2} \frac{\partial^{2}\left(\Phi^{T}\left(\sigma_{T}^{\mathrm{LV}}\right)^{2} S_{T}^{2}\right)}{\partial S_{T}^{2}}+\left(r_{T}^{d}-r_{T}^{f}\right) \frac{\partial\left(\Phi^{T} S_{T}\right)}{\partial S_{T}} \\
& -\frac{1}{2} \frac{\partial^{2}\left(\Phi\left(\sigma_{T}^{d}\right)^{2}\right)}{\partial\left(r_{T}^{d}\right)^{2}}+\frac{\partial\left(\Phi^{T} \alpha_{T}^{d}\right)}{\partial r_{T}^{d}}-\frac{1}{2} \frac{\partial^{2}\left(\Phi^{T}\left(\sigma_{T}^{f}\right)^{2}\right)}{\partial\left(r_{T}^{f}\right)^{2}}+\frac{\partial\left(\Phi^{T} \alpha_{T}^{f}\right)}{\partial r_{T}^{f}} \\
& -\frac{\partial^{2}\left(\Phi^{T} \rho^{S d} S_{T} \sigma_{T}^{\mathrm{LV}} \sigma_{T}^{d}\right)}{\partial S_{T} \partial r_{T}^{d}}-\frac{\partial^{2}\left(\Phi^{T} \rho^{S f} S_{T} \sigma_{T}^{\mathrm{LV}} \sigma_{T}^{f}\right)}{\partial S_{T} \partial r_{T}^{f}}-\frac{\partial^{2}\left(\Phi^{T} \rho^{d f} \sigma_{T}^{d} \sigma_{T}^{f}\right)}{\partial r_{T}^{d} \partial r_{T}^{f}} .
\end{aligned}
$$

\subsection{Extended Dupire formula}

\subsubsection{Call price surface formulation}

We integrate (2.12) over the entire ranges of $r_{T}^{d}$ and $r_{T}^{f}$. As before, as its arguments approach their limits the probability distribution function $\Phi^{T}$ and its derivatives go to zero fast enough to make the boundary terms vanish, and we obtain,

$$
\begin{aligned}
0= & \frac{\partial q^{T}}{\partial T}+\iint \Phi^{T}\left(r_{T}^{d}-f^{d}(0, T)\right) d r_{T}^{d} d r_{T}^{f}-\frac{1}{2} \frac{\partial^{2}\left(q^{T}\left(\sigma_{T}^{\mathrm{LV}}\right)^{2} S_{T}^{2}\right)}{\partial S_{T}^{2}} \\
& +\frac{\partial}{\partial S_{T}}\left(\iint\left(r_{T}^{d}-r_{T}^{f}\right) \Phi^{T} S_{T} d r_{T}^{d} d r_{T}^{f}\right) .
\end{aligned}
$$

The time zero value of a European vanilla call option $C$ with strike $K$, which pays off $\max \left(S_{T}-K, 0\right)$ at time $T$ is given by

$$
C=P^{d}(0, T) \mathbf{E}^{\mathbb{Q}^{\mathrm{T}}}\left[\left(S_{T}-K\right) \mathbb{1}_{S_{T}>K}\right]=P^{d}(0, T) \iiint_{K}^{\infty}\left(S_{T}-K\right) \Phi^{T} d S_{T} d r_{T}^{d} d r_{T}^{f}
$$

We compute the first two derivatives of the call price with respect to strike $K$,

$$
\begin{aligned}
\frac{\partial C}{\partial K} & =P^{d}(0, T) \iint\left[-\left.\left(S_{T}-K\right) \Phi^{T}\right|_{S_{T}=K} ^{\infty}-\int_{K}^{\infty} \Phi^{T} d S_{T}\right] d r_{T}^{d} d r_{T}^{f} \\
& =-P^{d}(0, T) \iiint_{K}^{\infty} \Phi^{T} d S_{T} d r_{T}^{d} d r_{T}^{f}=-P^{d}(0, T) \mathbf{E}^{\mathbb{Q}^{T}}\left[\mathbb{1}_{S_{T}>K}\right] \\
\frac{\partial^{2} C}{\partial K^{2}} & =P^{d}(0, T) \iint \Phi^{T}\left(K, r_{T}^{d}, r_{T}^{f}, T\right) d r_{T}^{d} d r_{T}^{f}=P^{d}(0, T) q^{T}(K, T) .
\end{aligned}
$$

Next, we differentiate the call price with respect to time. Here we make use of (2.13) and integration by parts,

$$
\begin{aligned}
\frac{\partial C}{\partial T}= & \frac{\partial P^{d}(0, T)}{\partial T} \iiint_{K}^{\infty}\left(S_{T}-K\right) \Phi^{T} d S_{T} d r_{T}^{d} d r_{T}^{f} \\
& +P^{d}(0, T) \iiint_{K}^{\infty}\left(S_{T}-K\right) \frac{\partial \Phi^{T}}{\partial T} d S_{T} d r_{T}^{d} d r_{T}^{f}
\end{aligned}
$$




$$
\begin{gathered}
=-f^{d}(0, T) C+P^{d}(0, T) \int_{K}^{\infty}\left(S_{T}-K\right) \frac{\partial q^{T}}{\partial T} d S_{T} \\
=-f^{d}(0, T) C+P^{d}(0, T) \int_{K}^{\infty}\left(S_{T}-K\right)\left\{-\iint \Phi^{T}\left(r_{T}^{d}-f^{d}(0, T)\right) d r_{T}^{d} d r_{T}^{f}\right. \\
+\frac{1}{2} \frac{\partial^{2}\left(q^{T}\left(\sigma_{T}^{\mathrm{LV}}\right)^{2} S_{T}^{2}\right)}{\partial S_{T}^{2}} \\
\left.-\frac{\partial}{\partial S_{T}}\left(\iint\left(r_{T}^{d}-r_{T}^{f}\right) \Phi^{T} S_{T} d r_{T}^{d} d r_{T}^{f}\right)\right\} d S_{T} \\
=P^{d}(0, T) \iiint_{K}^{\infty} \Phi^{T}\left(K r_{T}^{d}-S_{T} r_{T}^{f}\right) d S_{T} d r_{T}^{d} d r_{T}^{f}-\left.\frac{1}{2} P^{d}(0, T) q^{T} S_{T}^{2}\left(\sigma_{T}^{\mathrm{LV}}\right)^{2}\right|_{S_{T}=K} ^{\infty} .
\end{gathered}
$$

Plugging (2.16) into this expression yields

$$
\frac{\partial C}{\partial T}=P^{d}(0, T) \mathbf{E}^{\mathbb{Q}^{\mathrm{T}}}\left[\left(K r_{T}^{d}-S_{T} r_{T}^{f}\right) \mathbb{1}_{S_{T}>K}\right]+\frac{1}{2} K^{2} \frac{\partial^{2} C}{\partial K^{2}}\left(\sigma_{T}^{\mathrm{LV}}\right)^{2} .
$$

Thus we arrive at the extended Dupire formula under stochastic rates

$$
\sigma^{\mathrm{LV}}(K, T)^{2}=\frac{\frac{\partial C}{\partial T}-P^{d}(0, T) \mathbf{E}^{\mathbb{Q}^{\mathrm{T}}}\left[\left(K r_{T}^{d}-S_{T} r_{T}^{f}\right) \mathbb{1}_{S_{T}>K}\right]}{\frac{1}{2} K^{2} \frac{\partial^{2} C}{\partial K^{2}}}
$$

This is an implicit formula, where the expectation on the right hand side depends on the local volatility $\sigma^{\mathrm{LV}}(K, T)$, and it can be evaluated through a fixed-point iteration scheme. There is no known method to compute the expectation above analytically, yet it can be evaluated by numerical methods such as Monte Carlo or finite differences. Note also that the term with the expectation corresponds to the price of an option with maturity $T$ and payoff $\left(K r_{T}^{d}-S_{T} r_{T}^{f}\right) \mathbb{1}_{S_{T}>K}$.

Single stochastic rate limit In the limit where the foreign rates $r_{T}^{f}$ are deterministic, this equation becomes (see [5] for an alternative derivation)

$$
\sigma^{\mathrm{LV}}(K, T)^{2}=\frac{\frac{\partial C}{\partial T}-P^{d}(0, T) K \mathbf{E}^{\mathbb{Q}^{T}}\left[r_{T}^{d} \mathbb{1}_{S_{T}>K}\right]+P^{d}(0, T) r_{T}^{f} \mathbf{E}^{\mathbb{Q}^{T}}\left[S_{T} \mathbb{1}_{S_{T}>K}\right]}{\frac{1}{2} K^{2} \frac{\partial^{2} C}{\partial K^{2}}} .
$$

The second expectation in the numerator can be evaluated using (2.14) and (2.15)

$$
P^{d}(0, T) r_{T}^{f} \mathbf{E}^{\mathbb{Q}^{T}}\left[S_{T} \mathbb{1}_{S_{T}>K}\right]=r_{T}^{f}\left[C+P^{d}(0, T) K \mathbf{E}^{\mathbb{Q}^{T}}\left[\mathbb{1}_{S_{T}>K}\right]\right]=r_{T}^{f}\left[C-K \frac{\partial C}{\partial K}\right],
$$

which reduces (2.19) to

$$
\sigma^{\mathrm{LV}}(K, T)^{2}=\frac{\frac{\partial C}{\partial T}-P^{d}(0, T) K \mathbf{E}^{\mathbb{Q}^{\mathrm{T}}}\left[r_{T}^{d} \mathbb{1}_{S_{T}>K}\right]+r_{T}^{f}\left[C-K \frac{\partial C}{\partial K}\right]}{\frac{1}{2} K^{2} \frac{\partial^{2} C}{\partial K^{2}}}
$$


Deterministic rates limit In the limit where both the domestic rates $r_{T}^{d}$ and the foreign rates $r_{T}^{f}$ are deterministic, one can evaluate the expectation in the above numerator using (2.15)

$$
-P^{d}(0, T) \mathbf{E}^{\mathbb{Q}^{T}}\left[\mathbb{1}_{S_{T}>K}\right]=\frac{\partial C}{\partial K} .
$$

This allows us to reproduce the standard Dupire formula,

$$
\sigma^{\mathrm{LV}}(K, T)^{2}=\frac{\frac{\partial C}{\partial T}+\left(r_{T}^{d}-r_{T}^{f}\right) K \frac{\partial C}{\partial K}+r_{T}^{f} C}{\frac{1}{2} K^{2} \frac{\partial^{2} C}{\partial K^{2}}} .
$$

\section{Generalized Local Volatility Model}

\subsection{Model Setup}

In (2.1) we considered a standard local volatility process of a particular form. Namely it is geometric and the drift term is a linear combination of two stochastic rates, each modeled by a single factor process. Here we relax these constraints and study the following general model with drift and diffusion functions that allow arbitrary number of stochastic factors.

It is constructive to write down this SDE system in terms of $N$ independent Brownian motions under the domestic risk neutral measure $\mathbf{E}^{\mathbb{Q}^{\text {DRN }}}$,

$$
\begin{aligned}
d S_{t} & =\mu(\omega, t) d t+L\left(S_{t}, t\right) \sum_{k=1}^{N} \hat{\sigma}_{k}^{S}(\omega, t) d \hat{W}_{t}^{k}, \\
d y_{t}^{j} & =\mu^{j}(\omega, t) d t+\sum_{k=1}^{N} \hat{\sigma}_{k}^{j}(\omega, t) d \hat{W}_{t}^{k}, \\
d\left\langle\hat{W}^{k}, \hat{W}^{l}\right\rangle_{t} & =\delta^{k l} d t,
\end{aligned}
$$

where $\mu, \hat{\sigma}_{k}^{S}, \mu^{j}$, and $\hat{\sigma}_{k}^{j}$ are bounded functions of a general set of stochastic factors $\omega$. $Y_{t} \equiv\left(y_{t}^{1}, \ldots, y_{t}^{M}\right)$ is the set of additional Itô processes in the SDE system for which we do not assume any special form other than the above. $L\left(S_{t}, t\right)$ is the local volatility or leverage function we want to compute. In this setup, we observe that the correlation structure of the underlying assets is absorbed into the functions $\hat{\sigma}_{k}^{S}$ and $\hat{\sigma}_{k}^{j}$. The correlations themselves can be Itô processes, in which case they are assigned to particular $y_{t}^{j}$ s. The (domestic) discount factor $D_{t}$ is an adapted function of $Y_{t}$; yet in general we do not assume a particular mapping 2 .

The SDE system can also be written in terms of correlated Brownian motions split into

\footnotetext{
${ }^{2}$ In the foreign exchange setting of Section $2, r_{t}^{d}$ and $r_{t}^{f}$ are each direct components of $Y_{t}$. We will return to this particular case in Section 3.4. As another example, in case $r_{t}^{d}$ follows a multi-factor short rate model, it can be written as a function of the factors that are a subset of $Y_{t}$.
} 
those driving the processes of $S_{t}$ and $Y_{t}$ separately, with $N=N_{S}+N_{Y}$, as

$$
\begin{aligned}
& d S_{t}=\mu(\omega, t) d t+L\left(S_{t}, t\right) \sum_{k=1}^{N_{S}} \sigma_{k}^{S}(\omega, t) d W_{t}^{S k}, \\
& d y_{t}^{j}=\mu^{j}(\omega, t) d t+\sum_{k=1}^{N_{Y}} \sigma_{k}^{j}(\omega, t) d W_{t}^{Y k} .
\end{aligned}
$$

\subsection{Fokker-Planck Equation}

Following the same methodology from Section 2.2, omitting repetitive parts of the computation, we derive the corresponding Fokker-Planck equation. We compute the time derivative of the discounted value of a twice differentiable test function $V_{T}=V\left(S_{T}, Y_{T}\right)$ as

$$
\begin{aligned}
\frac{\partial \mathbf{E}^{\mathbb{Q}^{\mathrm{DRN}}}\left[D_{T} V_{T}\right]}{\partial T}=\mathbf{E}^{\mathbb{Q}^{\mathrm{DRN}}}\left[D_{T}(\right. & -r_{T}^{d} V_{T}+\frac{1}{2} L_{T}^{2} \bar{\sigma}_{T}^{2} \frac{\partial^{2} V_{T}}{\partial S_{T}^{2}}+\mu_{T} \frac{\partial V_{T}}{\partial S_{T}} \\
& \left.\left.+ \text { terms involving } Y_{T} \text { derivatives of } V_{T}\right)\right],
\end{aligned}
$$

where $r_{T}^{d} \equiv-\frac{1}{D_{T}} \frac{\partial D_{T}}{\partial T}$ is not assumed to follow a particular stochastic process, we defined

$$
\bar{\sigma}_{T}^{2} \equiv \sum_{l, m=1}^{N_{S}}\left(\sigma_{l}^{S} \rho_{l m}^{S} \sigma_{m}^{S}\right)=\sum_{k=1}^{N}\left(\hat{\sigma}_{k}^{S}\right)^{2},
$$

and to keep the notation compact we denoted $\mu_{T}=\mu(\omega, T)$ and $L_{T}=L\left(S_{T}, T\right)$. Here $\rho_{l m}^{S}$ denotes the correlation function between the Brownian motions $W_{t}^{S l}$, i.e. $d\left\langle W^{S l}, W^{S m}\right\rangle_{t}=$ $\rho_{l m}^{S} d t$. Combining this with the expression for the time derivative of the discounted value of the test function in $T$-forward measure $\mathbb{Q}^{\mathrm{T}}$ yields

$$
\begin{aligned}
& 0= \frac{\partial P^{d}(0, T)}{\partial T} \iint V_{T} \Phi^{T} d S_{T} d Y_{T}+P^{d}(0, T) \iint V_{T} \frac{\partial \Phi^{T}}{\partial T} d S_{T} d Y_{T} \\
&+P^{d}(0, T) \iint \Phi^{T}\left[r_{T}^{d} V_{T}-\frac{1}{2} L_{T}^{2} \bar{\sigma}_{T}^{2} \frac{\partial^{2} V_{T}}{\partial S_{T}^{2}}-\mu_{T} \frac{\partial V_{T}}{\partial S_{T}}\right. \\
&\left.+ \text { terms involving } Y_{T} \text { derivatives of } V_{T}\right] d S_{T} d Y_{T}
\end{aligned}
$$

where $\Phi^{T}\left(S_{T}, Y_{T}, T\right)$ is the $T$-forward measure probability density, with the corresponding marginal density $q^{T}\left(S_{T}, T\right)=\int \Phi^{T}\left(S_{T}, Y_{T}, T\right) d Y_{T}$, which can be used to formulate the derivatives of the call option price with respect to strike, analogous to (2.15) and (2.16) as

$$
\begin{aligned}
\frac{\partial C}{\partial K} & =-P^{d}(0, T) \mathbf{E}^{\mathbb{Q}^{T}}\left[\mathbb{1}_{S_{T}>K}\right], \\
\frac{\partial^{2} C}{\partial K^{2}} & =P^{d}(0, T) q^{T}(K, T) .
\end{aligned}
$$

Moreover, to elucidate the notation, we make a note that the integrals along the stochastic factors $Y_{T}$

$$
\int f(\cdot) d Y_{T} \equiv \int \ldots \int f(\cdot) d y_{T}^{1} d y_{T}^{2} \ldots
$$


are taken over their entire domains.

Since $\frac{\partial P^{d}(0, T)}{\partial T}=-f^{d}(0, T) P^{d}(0, T),(3.3)$ becomes

$$
\begin{aligned}
0=\iint\left[V_{T} \frac{\partial \Phi^{T}}{\partial T}+\Phi^{T}\right. & \left\{\left(r_{T}^{d}-f^{d}(0, T)\right) V_{T}-\frac{1}{2} L_{T}^{2} \bar{\sigma}_{T}^{2} \frac{\partial^{2} V_{T}}{\partial S_{T}^{2}}-\mu_{T} \frac{\partial V_{T}}{\partial S_{T}}\right. \\
& \left.\left.+ \text { terms involving } Y_{T} \text { derivatives of } V_{T}\right\}\right] d S_{T} d Y_{T} .
\end{aligned}
$$

As before, we integrate by parts the above integrals to factor out $V_{T}$. This leads to the following Fokker-Planck equation,

$$
\begin{aligned}
0= & \frac{\partial \Phi^{T}}{\partial T}+\Phi^{T}\left(r_{T}^{d}-f^{d}(0, T)\right)-\frac{1}{2} \frac{\partial^{2}\left(\Phi^{T} L_{T}^{2} \bar{\sigma}_{T}^{2}\right)}{\partial S_{T}^{2}}+\frac{\partial\left(\Phi^{T} \mu_{T}\right)}{\partial S_{T}} \\
& + \text { terms involving } Y_{T} \text { derivatives of } \Phi^{T} .
\end{aligned}
$$

\subsection{Generalized Dupire formula}

As in Section 2.3.1, we integrate the Fokker-Planck equation (3.4) over the entire ranges of $Y_{T}$. The probability distribution function $\Phi^{T}$ goes to zero fast enough as its arguments approach their limits, making the boundary terms that involve the $Y_{T}$ derivatives vanish,

$$
\begin{aligned}
0= & \frac{\partial q^{T}}{\partial T}+\int \Phi^{T}\left(r_{T}^{d}-f^{d}(0, T)\right) d Y_{T}-\frac{1}{2} \frac{\partial^{2}}{\partial S_{T}^{2}}\left(L_{T}^{2} \int \Phi^{T} \bar{\sigma}_{T}^{2} d Y_{T}\right) \\
& +\frac{\partial}{\partial S_{T}}\left(\int \Phi^{T} \mu_{T} d Y_{T}\right) .
\end{aligned}
$$

At this point we note that the terms involving the correlation coefficients are all integrated out, therefore we conclude that the nature of the correlations will not have any impact on our result. Next we compute the time derivative of the price of a European vanilla call option $C$ with strike $K$. Here we make use of the definition of conditional expectation, $\Phi^{T}\left(Y, T \mid S_{T}=X\right) \equiv \frac{\Phi^{T}(X, Y, T)}{q^{T}(X, T)}$, as well as (3.5) and integration by parts,

$$
\begin{aligned}
& \frac{\partial C}{\partial T}= \frac{\partial P^{d}(0, T)}{\partial T} \iint_{K}^{\infty}\left(S_{T}-K\right) \Phi^{T} d S_{T} d Y_{T} \\
&=-f^{d}(0, T) C+P^{d}(0, T) \iint_{K}^{\infty}\left(S_{T}-K\right) \frac{\partial \Phi^{T}}{\partial T} d S_{T} d Y_{T} \\
&=-f^{d}(0, T) C+P^{d}(0, T) \int_{K}^{\infty}\left(S_{T}-K\right) \frac{\partial q^{T}}{\partial T} d S_{T} \\
&-\int \Phi^{T}\left(r_{T}^{d}-f^{d}(0, T)\right) d Y_{T} \\
&+\frac{1}{2} \frac{\partial^{2}}{\partial S_{T}^{2}}\left(L_{T}^{2} \int \Phi^{T} \bar{\sigma}_{T}^{2} d Y_{T}\right) \\
&\left.\quad-\frac{\partial}{\partial S_{T}}\left(\int \Phi^{T} \mu_{T} d Y_{T}\right)\right\} d S_{T} \\
&=P^{d}(0, T) \iint_{K}^{\infty} \Phi^{T}\left[\mu_{T}-\left(S_{T}-K\right) r_{T}^{d}\right] d S_{T} d Y_{T} \\
&+\frac{1}{2} P^{d}(0, T) q^{T}(K, T) L(K, T)^{2} \iint \Phi^{T}\left(Y_{T}, T \mid S_{T}=K\right) \bar{\sigma}_{T}^{2} d Y_{T}
\end{aligned}
$$




$$
=P^{d}(0, T) \mathbf{E}^{\mathbb{Q}^{T}}\left[\left\{\mu_{T}-\left(S_{T}-K\right) r_{T}^{d}\right\} \mathbb{1}_{S_{T}>K}\right]+\frac{1}{2} L(K, T)^{2} \frac{\partial^{2} C}{\partial K^{2}} \mathbf{E}^{\mathbb{Q}^{T}}\left[\bar{\sigma}_{T}^{2} \mid S_{T}=K\right] .
$$

This gives us the generalized form of the Dupire formula,

$$
L(K, T)^{2}=\frac{\frac{\partial C}{\partial T}-P^{d}(0, T) \mathbf{E}^{\mathbb{Q}^{T}}\left[\left\{\mu_{T}-\left(S_{T}-K\right) r_{T}^{d}\right\} \mathbb{1}_{S_{T}>K}\right]}{\frac{1}{2} \frac{\partial^{2} C}{\partial K^{2}} \mathbf{E}^{\mathbb{Q}^{T}}\left[\bar{\sigma}_{T}^{2} \mid S_{T}=K\right]} .
$$

As was the case with the extended Dupire formula (2.18), this is an implicit equation, where the expectations on the right hand side depend on the leverage function $L(K, T)$, and it can be evaluated through a fixed-point iteration scheme.

\subsection{Examples}

The generalized Dupire formula (3.6) applies to a wide range of models.

\subsubsection{Simple Models}

For simplicity, we consider the underlier $S_{t}$ to be driven by a single Brownian motion $\left(N_{S}=1\right)$ in this section,

$$
d S_{t}=\mu(\omega, t) d t+L_{s}\left(S_{t}, t\right) \bar{\sigma}(\omega, t) d W_{t}^{S},
$$

where $L_{s}$ denotes the leverage function of the simplified model, to distinguish it from the generalized model.

The special case of this model with $\bar{\sigma}=S_{t}$ is of special interest where the SDE becomes a simple local volatility model. In this case (3.6) becomes

$$
\sigma^{\mathrm{LV}}(K, T)^{2}=\frac{\frac{\partial C}{\partial T}-P^{d}(0, T) \mathbf{E}^{\mathbb{Q}^{\mathrm{T}}}\left[\left\{\mu_{T}-\left(S_{T}-K\right) r_{T}^{d}\right\} \mathbb{1}_{S_{T}>K}\right]}{\frac{1}{2} K^{2} \frac{\partial^{2} C}{\partial K^{2}}} .
$$

Comparison of (3.6) with (3.7) gives us the following relationship between the generalized model and its corresponding simple local volatility simplification,

$$
\sigma^{\mathrm{LV}}(K, T)^{2} K^{2}=L(K, T)^{2} \mathbf{E}^{\mathbb{Q}^{\mathrm{T}}}\left[\bar{\sigma}^{2} \mid S_{T}=K\right] .
$$

To recover the simpler FX local volatility model with two stochastic rates $\left(Y_{t}=r_{t}^{d}, r_{t}^{f}\right)$ from Section 2.1, one can set $\mu_{t}=\left(r_{t}^{d}-r_{t}^{f}\right) S_{t}$ and $\bar{\sigma}=S_{t}$. In this case it is straightforward to show that (3.6) reduces to (2.18).

\subsubsection{Stochastic Local Volatility}

An extension of the simple model is the stochastic local volatility (SLV) model with $\bar{\sigma}=$ $S_{t} \sqrt{U_{t}}$ where $U_{t}$ is the variance process,

$$
d U_{t}=\mu^{U}\left(U_{t}, t\right) d t+\sigma^{U}\left(U_{t}, t\right) d W_{t}^{U},
$$

which is typically chosen to fit certain options or ranges or aspects of the price, and the leverage function $L\left(S_{t}, T\right)$ serves as a correction that ensures that all vanilla options are 
repriced over the full calibration range. A common choice is to use a Cox-Ingersoll-Ross (CIR) process [13] in which case in the context of FX derivatives the SDE system becomes [8, 14],

$$
\begin{aligned}
& d S_{t}=\left(r_{t}^{d}-r_{t}^{f}\right) S_{t} d t+L_{s}\left(S_{t}, t\right) S_{t} \sqrt{U_{t}} d W_{t}^{S(\mathrm{DRN})} \\
& d U_{t}=\kappa\left(\theta-U_{t}\right) d t+\xi \sqrt{U_{t}} d W_{t}^{U(\mathrm{DRN})}
\end{aligned}
$$

where mean reversion speed $\kappa$, long term mean $\theta$, and vol-of-vol $\xi$ are possibly timedependent CIR parameters.

For this model, the generalized Dupire formula (3.6) simplifies to

$$
L_{s}(K, T)^{2}=\frac{\frac{\partial C}{\partial T}-P^{d}(0, T) \mathbf{E}^{\mathbb{Q}^{\mathrm{T}}}\left[\left(K r_{T}^{d}-S_{T} r_{T}^{f}\right) \mathbb{1}_{S_{T}>K}\right]}{\frac{1}{2} K^{2} \frac{\partial^{2} C}{\partial K^{2}} \mathbf{E}^{\mathbb{Q}^{T}}\left[U_{T} \mid S_{T}=K\right]} .
$$

Here we emphasize that the above equation assumes only the particular form of the SDE for the underlier $S_{t}$, and is not restricted to the case where the SDE for the variance $U_{t}$ is of type CIR.

Comparing (2.18) to (3.10) allows us to write the relationship between the local volatility function of the simple local volatility model (2.1) and the leverage function of the stochastic local volatility model (3.9) as

$$
\sigma^{\mathrm{LV}}(K, T)^{2}=L_{s}(K, T)^{2} \mathbf{E}^{\mathbb{Q}^{\mathrm{T}}}\left[U_{T} \mid S_{T}=K\right] .
$$

This relationship is reached independently from but is consistent with Gyöngy's finding [15] that links the set of stochastic processes $X_{t} \equiv\left\{X_{t}^{i}\right\}$ with Itô differentials

$$
d X_{t}^{m}=\alpha^{m}(t, \omega) d t+\sum_{n=1}^{N} \beta^{m n}(t, \omega) d \tilde{W}_{t}^{n}
$$

where $\alpha^{m}$ and $\beta^{m n}$ are bounded functions of a general set of stochastic factors $\omega$, which may include factors not contained in or related to $X_{t}$, and $\left\{\tilde{W}_{t}^{i}\right\}$ are Brownian motions under measure $\mathbb{P}$; to another set of stochastic processes $Z_{t} \equiv\left\{Z_{t}^{i}\right\}$ with deterministic coefficients $a^{m}$ and $b^{m n}$,

$$
d Z_{t}^{m}=a^{m}\left(t, Z_{t}\right) d t+\sum_{n=1}^{N} b^{m n}\left(t, Z_{t}\right) d \hat{W}_{t}^{n},
$$

where $\left\{\hat{W}_{t}^{i}\right\}$ are Brownian motions under measure $\mathbb{Q}$, in that the two sets of processes have the same marginal probability distribution, $X_{t}$ under $\mathbb{P}$ and of $Z_{t}$ under $\mathbb{Q}$, for every $t$ if

$$
\begin{aligned}
a^{m}(t, z) & =\mathbf{E}^{\mathbb{P}}\left[\alpha^{m}(t, \omega) \mid X_{t}=z\right], \\
\sum_{p=1}^{N} b^{m p}(t, z) b^{n p}(t, z) & =\mathbf{E}^{\mathbb{P}}\left[\sum_{p=1}^{N} \beta^{m p}(t, \omega) \beta^{n p}(t, \omega) \mid X_{t}=z\right],
\end{aligned}
$$

for all $m, n=1, \ldots, N$. 
Applying this to our example, the marginal distribution of the stochastic local volatility model (3.9) must be the same as the distribution of the simple local volatility model (2.1) if (3.11) holds. This implies that having computed the function $\sigma^{\mathrm{LV}}(K, T)$ for the simple local volatility model using (2.18), one can obtain the leverage function $L_{s}(K, T)$ of the stochastic local volatility model by evaluating the conditional expectation $\mathbf{E}^{\mathbb{Q}^{\mathrm{T}}}\left[U_{T} \mid S_{T}=K\right]$. One utilizes a numerical method such as multi-dimensional finite difference or Monte Carlo simulation to estimate this conditional expectation as there is no straightforward way to evaluate it analytically.

\section{Case study}

In this section we study FX local volatility model where the domestic and foreign rates are governed by stochastic processes. For the rates evolution we consider the Linear Gaussian Model, which we first briefly introduce.

\subsection{Linear Gaussian Model}

In the Linear Gaussian Model (LGM), as proposed by [16], the rate is driven by a single Markovian factor

$$
d x_{t}=\sigma_{t} d W_{t}^{N}, x_{0}=0,
$$

in the measure $\mathbb{Q}^{\mathrm{N}}$ defined by the numéraire

$$
N\left(t, x_{t}\right)=\frac{1}{P(0, t)} \exp \left[H_{t} x_{t}+\frac{1}{2} H_{t}^{2} \zeta_{t}\right]
$$

where

$$
\begin{aligned}
\zeta_{t} & =\int_{0}^{t} \sigma_{s}^{2} d s, \\
H_{t} & =\int_{0}^{t} h_{s} d s,
\end{aligned}
$$

and the model parameters $\sigma_{t}$ and $h_{t}$ are calibrated to the market quotes. The short rate is given by [17]

$$
r\left(t, x_{t}\right)=f(0, t)+h_{t} x_{t}+h_{t} H_{t} \zeta_{t},
$$

where the instantaneous forward rate $f(0, t)$ is computed from the zero coupon bond curve $P(0, t)$ as in (2.10).

The change to the risk neutral measure $\mathbb{Q}^{\mathrm{RN}}$, where the money market account is the numéraire, is derived as [16],

$$
d W_{t}^{(\mathrm{RN})}=d W_{t}^{N}+\sigma_{t} H_{t} d t,
$$

so that the Markovian factor evolves in this measure as

$$
d x_{t}=-\sigma_{t}^{2} H_{t} d t+\sigma_{t} d W_{t}^{(\mathrm{RN})} .
$$




\subsection{Local Volatility with LGM rates}

We consider a three-factor local volatility model for the FX rate $S$, where the domestic rate $r^{d}$ and foreign rate $r^{f}$ are governed by LGM processes, with time dependent parameters $\sigma^{d}, h^{d}$ and $\sigma^{f}, h^{f}$ respectively. The joint evolution of the three factors is given by

$$
\begin{aligned}
& d S_{t}=\left[r_{t}^{d}-r_{t}^{f}\right] S_{t} d t+\sigma^{\mathrm{LV}}\left(S_{t}, t\right) S_{t} d W_{t}^{S(\mathrm{DRN})}, \\
& d x_{t}^{d}=-\left(\sigma_{t}^{d}\right)^{2} H_{t}^{d} d t+\sigma_{t}^{d} d W_{t}^{d(\mathrm{DRN})} \\
& d x_{t}^{f}=-\left[\left(\sigma_{t}^{f}\right)^{2} H_{t}^{f}+\rho^{S f} \sigma_{t}^{f} \sigma^{\mathrm{LV}}\left(S_{t}, t\right)\right] d t+\sigma_{t}^{f} d W_{t}^{f(\mathrm{DRN})} .
\end{aligned}
$$

The coefficients of correlation between the Brownian motions $W_{t}^{S(\mathrm{DRN})}, W_{t}^{d(\mathrm{DRN})}, W_{t}^{f(\mathrm{DRN})}$, evolving under the domestic risk neutral measure $\mathbf{E}^{\mathbb{Q}^{D R N}}$, are given by the quadratic covariances

$$
\begin{aligned}
& d\left\langle W^{S(\mathrm{DRN})}, W^{d(\mathrm{DRN})}\right\rangle_{t}=\rho^{S d} d t, \\
& d\left\langle W^{S(\mathrm{DRN})}, W^{f(\mathrm{DRN})}\right\rangle_{t}=\rho^{S f} d t, \\
& d\left\langle W^{d(\mathrm{DRN})}, W^{f(\mathrm{DRN})}\right\rangle_{t}=\rho^{d f} d t .
\end{aligned}
$$

\subsection{Calibrating the local volatility surface}

The Radon-Nikodym derivative (2.4) allows us to transform the extended Dupire formula (2.18) to

$$
\sigma^{\mathrm{LV}}(K, T)^{2}=\frac{\frac{\partial C}{\partial T}-\mathbf{E}^{\mathbb{Q}^{\mathrm{DRN}}}\left[D_{T}\left(K r_{T}^{d}-S_{T} r_{T}^{f}\right) \mathbb{1}_{S_{T}>K}\right]}{\frac{1}{2} K^{2} \frac{\partial^{2} C}{\partial K^{2}}} .
$$

The expectation in the above expression can be estimated by Monte Carlo simulation.

We propose the following algorithm to calibrate the local volatility surface at time slices $t_{j}, j=1, \ldots, M$, by solving (4.2) in a fixed point iteration scheme:

1. Using the market implied volatility $\Sigma(K, t)$, generate a vanilla call option price surface $C(K, t)$ interpolator (or a total implied variance surface $w(y, t)$ interpolator).

2. For the first time slice $T=t_{1}>0$, (a) in the first iteration evaluate the deterministic equation (2.21) to compute the FX local volatilities for a predetermined range of strikes. This step requires no Monte Carlo simulation. (b) in the subsequent iterations, simulate the SDE system (4.1) up to time $t_{1}$ using the local vol values from previous iteration. Compute the Monte Carlo estimate for the expectation $\mathbf{E}^{\mathbb{Q}^{\mathrm{DRN}}}\left[D_{T}\left(K r_{T}^{d}-S_{T} r_{T}^{f}\right) \mathbb{1}_{S_{T}>K}\right]$ appearing in (4.2) for the same set of strikes. Update local vol values with this equation.

3. For each of the subsequent time slices $T=t_{j}, j>1$, simulate the SDE system (4.1) up to time $t_{j}$, where (a) in the first iteration use the local vol values from time slice $t_{j-1}$ for time slice $t_{j}$, (b) in the subsequent iterations use local vol values from previous iteration for time slice $t_{j}$; and linearly interpolate the local volatility values between 
$t_{j-1}$ and $t_{j}$ slices for simulation times between the slices. Compute the Monte Carlo estimate for the expectation $\mathbf{E}^{\mathbb{Q}^{\mathrm{DRN}}}\left[D_{T}\left(K r_{T}^{d}-S_{T} r_{T}^{f}\right) \mathbb{1}_{S_{T}>K}\right]$ for a predetermined range of strikes. Update local vol values with the extended Dupire equation (4.2).

\subsection{Implementation and Results}

The EUR-USD market data as of 2021-09-30 we use in our analysis contains the EURUSD spot FX rate $S_{0}$, the EUR-USD implied volatility surface representing market quotes of vanilla option instruments on FX rate $S_{t}$, the discount curves $P^{d}(0, t), P^{f}(0, t)$ for the domestic rate $r_{t}^{d}$ and the foreign rate $r_{t}^{f}$, respectively. The coefficients of correlation are given by $\rho^{S d}=0.059, \rho^{S f}=0.031, \rho^{d f}=0.255$. For LGM model parameters, we use $\sigma_{t}^{d}=\sigma_{t}^{f}=0.01$, and $h_{t}^{d}=h_{t}^{f}=1$.

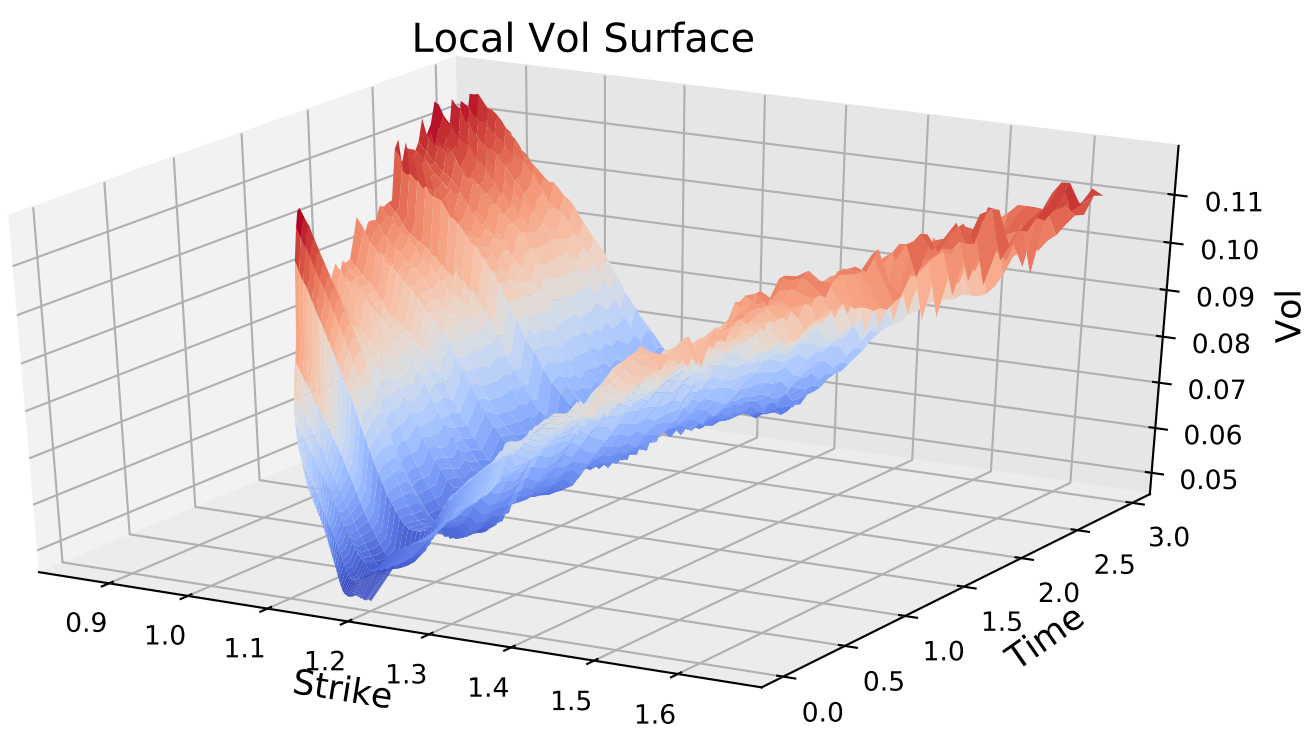

Figure 4.1: Calibrated EUR-USD local volatility

We calibrate the local volatility at time slices $t_{j}=0.05,0.1, \ldots, 3.0$. At each time slice, we select the strike grid $K_{j}^{l}$ to span the strike range $\left[F_{t_{j}} e^{-3 \Sigma\left(F_{t_{j}}, t_{j}\right) \sqrt{t_{j}}}, F_{t_{j}} e^{3 \Sigma\left(F_{t_{j}}, t_{j}\right) \sqrt{t_{j}}}\right]$ uniformly spaced in log-moneyness, where $F_{t_{j}}=S_{0} \frac{P^{f}\left(0, t_{j}\right)}{P^{d}\left(0, t_{j}\right)}$ is the forward asset price, and $\Sigma\left(F_{t_{j}}, t_{j}\right)$ is the at-the-money-forward implied volatility.

The setup for simulation is as follows. The simulation time step size is set to 0.004 years, so that the simulation times are $t_{i}=0.004,0.008, \ldots$ The SDE system (4.1) is simulated up to calibration time $t_{j}$ in forward Euler scheme,

$$
\begin{aligned}
& \Delta S_{i+1}=\left[r_{i}^{d}-r_{i}^{f}\right] S_{i} \Delta t_{i}+\sigma^{\mathrm{LV}}\left(S_{i}, t_{i}\right) S_{i} \sqrt{\Delta t_{i}} Z_{i}^{S}, \\
& \Delta x_{i+1}^{d}=-\left(\sigma_{t_{i}}^{d}\right)^{2} H_{t_{i}}^{d} \Delta t_{i}+\sigma_{t_{i}}^{d} \sqrt{\Delta t_{i}} Z_{i}^{d}, \\
& \Delta x_{i+1}^{f}=-\left[\left(\sigma_{t_{i}}^{f}\right)^{2} H_{t_{i}}^{f}+\rho^{S f} \sigma_{t_{i}}^{f} \sigma^{\mathrm{LV}}\left(S_{i}, t_{i}\right)\right] \Delta t_{i}+\sigma_{t_{i}}^{f} \sqrt{\Delta t_{i}} Z_{i}^{f},
\end{aligned}
$$



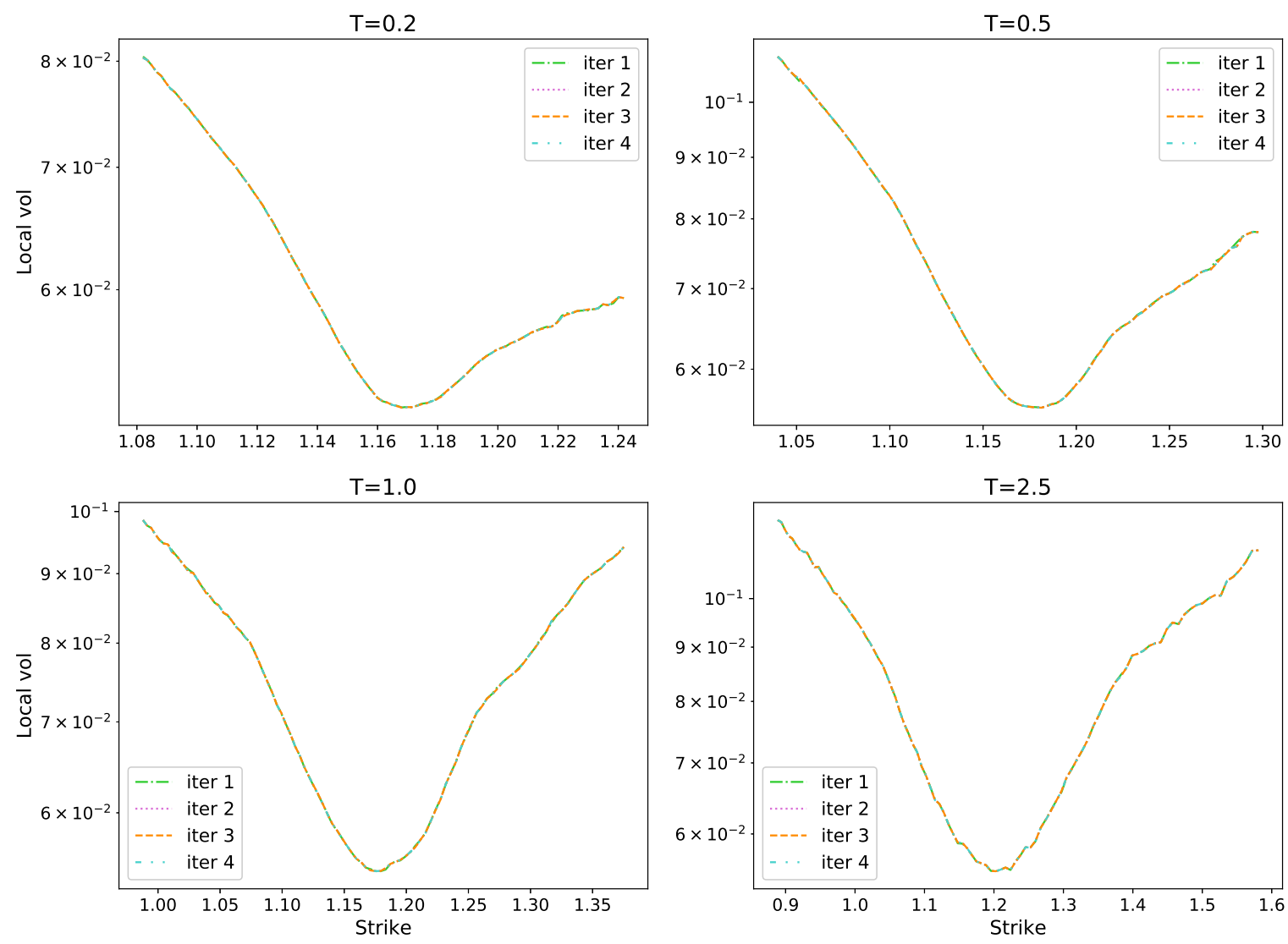

Figure 4.2: Calibrated local volatility values at several time slices after each iteration

where $\Delta t_{i}=t_{i+1}-t_{i}$. The random numbers $\left(Z_{i}^{S}, Z_{i}^{d}, Z_{i}^{f}\right)$ are drawn from the normal distribution $N(0, \Sigma)$, where

$$
\Sigma=\left(\begin{array}{ccc}
1 & \rho^{S d} & \rho^{S f} \\
\rho^{S d} & 1 & \rho^{d f} \\
\rho^{S f} & \rho^{d f} & 1
\end{array}\right)
$$

using Cholesky decomposition.

We simulate 1000 paths and their antithetic conjugate to compute the Monte Carlo average of $\left[D_{t_{j}}\left(K_{t_{j}}^{l} r_{t_{j}}^{d}-S_{t_{j}} r_{t_{j}}^{f}\right) \mathbb{1}_{S_{t_{j}}>K_{t_{j}}^{l}}\right]$ for all $K_{t_{j}}^{l}$ in the strike grid at time slice $t_{j}$, which we plug in to the expectation in (4.2) to compute $\sigma^{\mathrm{LV}}\left(K_{t_{j}}^{l}, t_{j}\right)$. The number of iterations at each time slice is set to 4 .

In Figure 4.1 the surface plot can be seen as a visual evidence that the local volatility calibration completed smoothly. To see the impact of successive iterations, we plot the local volatility curve after each iteration at time slices $t_{j}=0.2,0.5,1.0,2.5$ in Figure 4.2, As can be seen in the plots, the successive iterations do not result in a visual change in the calibrated local volatility values. In Figure 4.3 we see that the relative differences in local volatility between successive iterations shrinks fast, which shows that the impact of each additional iteration is relatively low. As the computational time scales linearly with 

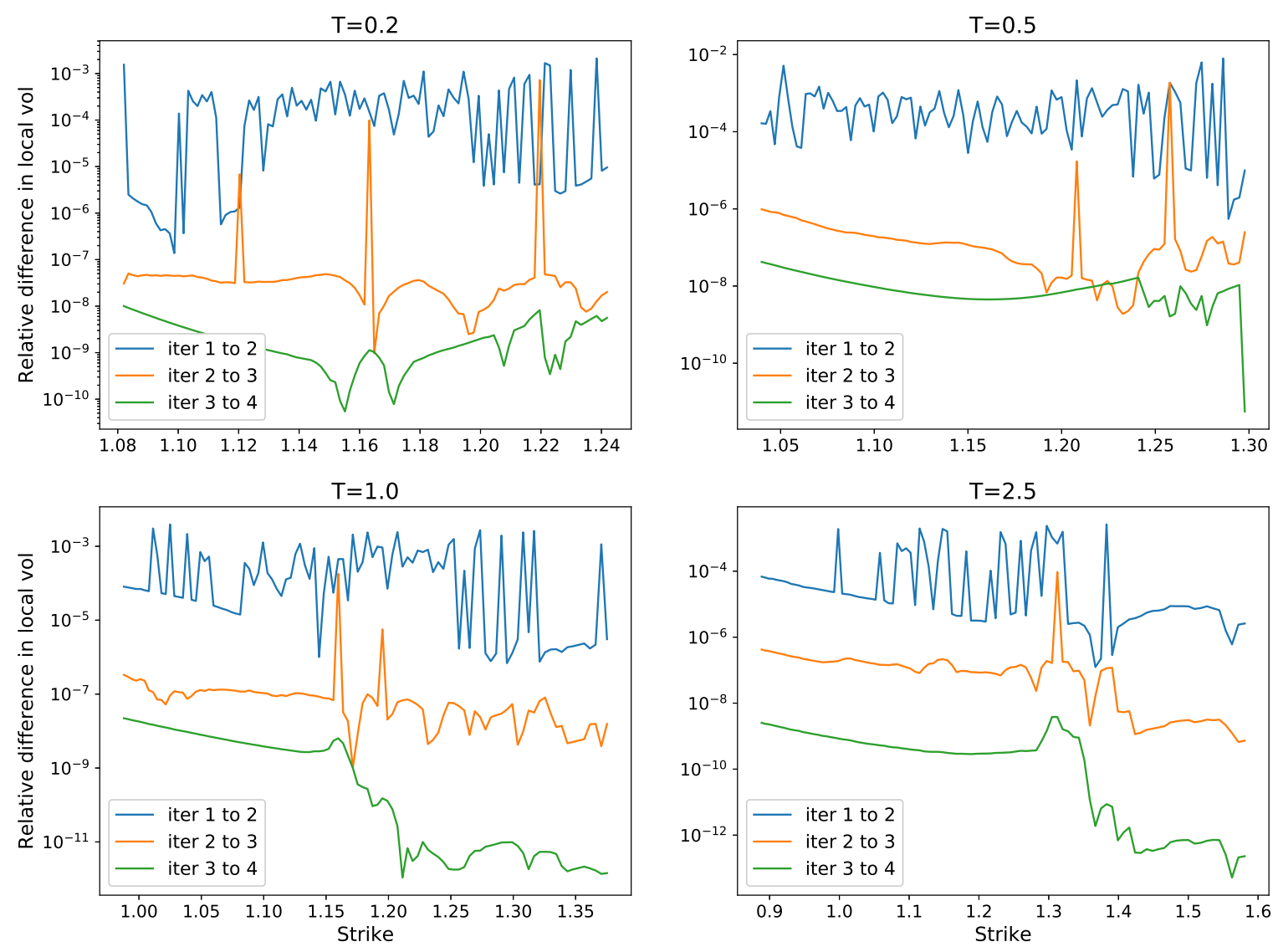

Figure 4.3: The local volatility update $\frac{\left|\sigma_{I+1}^{\mathrm{LV}}-\sigma_{I}^{\mathrm{LV}}\right|}{\sigma_{I}^{\mathrm{LV}}}$ after iterations $I=1,2,3$, at several time slices

the number of iterations, we conclude that convergence is achieved by as low as a single iteration.

Finally, to validate the quality of the calibrated volatility surface, we run a successive simulation of (4.3), with the same setup we used for calibration, to price a range of vanilla call options with expiries $T=0.2,0.5,1.0,2.5$. Figure 4.4 shows that the Monte Carlo prices match well with the market prices computed from the input implied volatility surface. The differences seem to be within two Monte Carlo errors.

\section{Discussion}

We derived the extension of the Dupire formula where the drift of the local volatility model is given as a difference of two stochastic processes of general form. The extended formula (2.18) can be used to calibrate local volatility models with stochastic rates of this structure. We further studied a general local volatility model where drift and diffusion are functions of arbitrary number of stochastic factors, with the simple assumption that the discount factor and the asset volatility are adapted functions of the Itô processes in the SDE system. The resulting generalized Dupire formula (3.6) can be used to calibrate a range of models, 

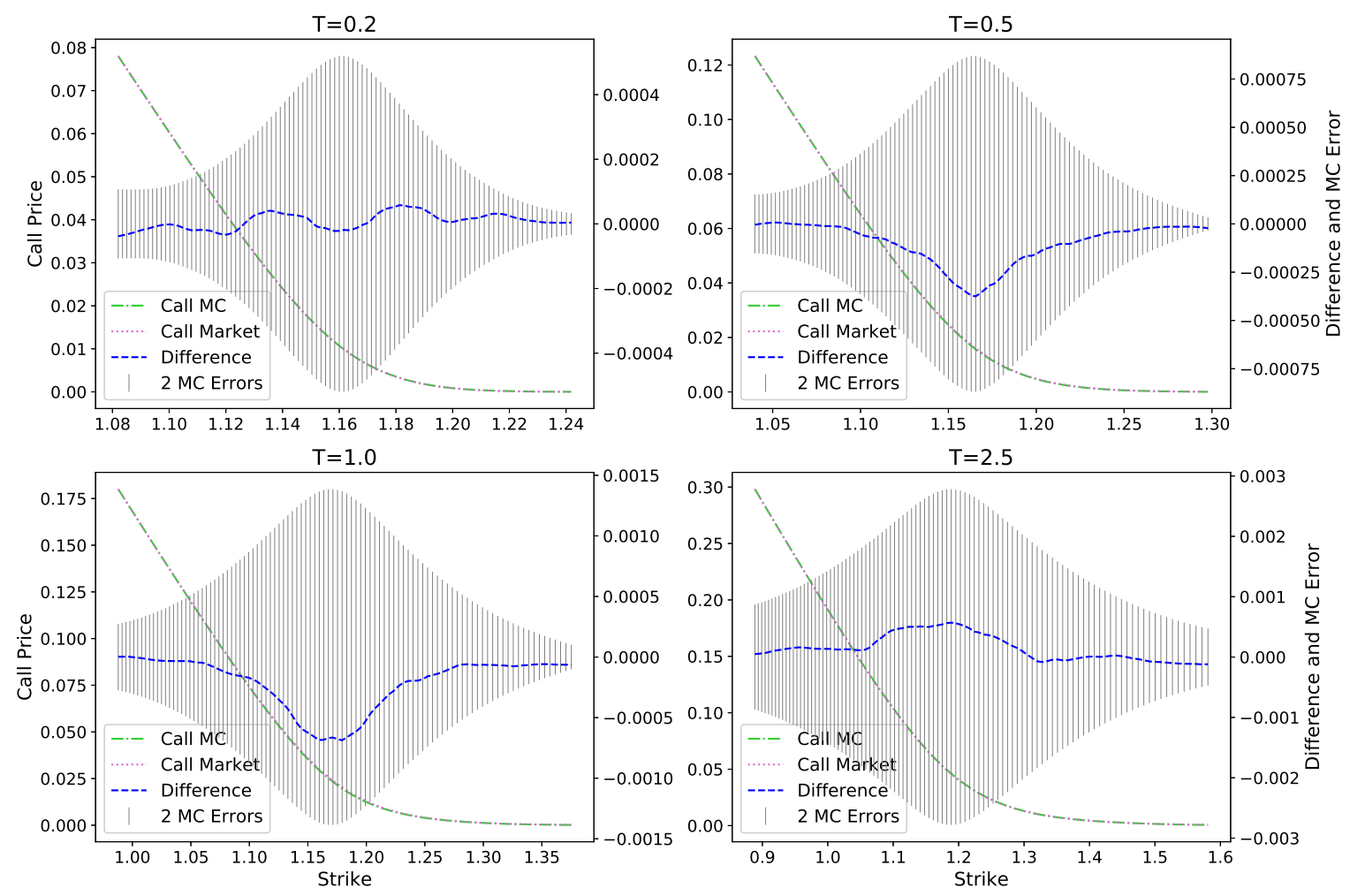

Figure 4.4: Comparing Monte Carlo prices to market prices of vanilla call options at several time slices. The differences are within two Monte Carlo error bounds.

including stochastic local volatility models with stochastic interest rates. Both of these equations are given in implicit form, where the expectations on the right hand side depend on the local volatility (leverage) function. Such equations can be solved numerically by fixed-point iteration schemes. The expectations appearing in these equations have no known analytical solutions, yet they can be evaluated by numerical methods such as Monte Carlo or finite differences. We presented a case study to demonstrate a calibration scheme by Monte Carlo simulation. In the study we iteratively calibrated a local volatility model subject to stochastic dometic and foreign interest rates, which were chosen to follow an LGM process. The results show that the calibration is achieved after a single iteration by a relatively low number of simulation paths, and that the calibrated local volatility surface recovers market prices accurately.

\section{A Total implied variance surface formulation}

Quotes for various European call options with a range of strikes and maturities are required for the evaluation of the Dupire formula (2.21) or the extended Dupire formula (2.18) to create a local volatility surface. In practice, one can create a call price surface interpolator to evaluate the call price and its derivatives in these equations along the grid where the local volatility surface is being constructed. However the method for interpolation while 
evaluating the Dupire formula is a concern as the interpolated values might introduce arbitrage to the model. One way to address this problem is to construct a Black-Scholes total implied variance surface and interpolate that instead. As a matter of fact, practitioners typically work with market data that is in the form of parametrized or dense implied volatility surfaces that are calibrated with such penalty functions that aim to avoid or at least to minimize arbitrage. The absence of calendar spread arbitrage implies that the total implied variance surface is a monotonically increasing function of time [18, 19]. By construction, interpolating the total implied variance surface and using these values in the Dupire formula avoids calendar spread arbitrage. In this section we derive the total implied variance parametrization of the extended Dupire formula.

The Black-Scholes European call option price function $C_{\mathrm{BS}}$ can be parametrized in terms of log-moneyness

$$
y(K, T)=\log \frac{K}{F_{T}},
$$

where $F_{T} \equiv S_{0} \frac{P^{f}(0, T)}{P^{d}(0, T)}$ is the forward price at time $T$, and the total implied variance

$$
w(y(K, T), T)=\Sigma(K, T)^{2} T
$$

as $[20$ ]

$$
C_{\mathrm{BS}}\left(P^{d}(0, T) F_{T}, y, w\right)=P^{d}(0, T) F_{T}\left(N\left(d_{1}\right)-e^{y} N\left(d_{2}\right)\right)
$$

with

$$
\begin{aligned}
& d_{1}=-y w^{-\frac{1}{2}}+\frac{1}{2} w^{\frac{1}{2}}, \\
& d_{2}=d_{1}-w^{\frac{1}{2}} .
\end{aligned}
$$

Here $\Sigma(K, T)$ is the market implied volatility at strike $K$ and maturity $T$, and $N(\cdot)$ is the standard Gaussian cumulative distribution function. Noting that both $C_{\mathrm{BS}}$ and $w$ depend on the strike $K$ indirectly through $y(K, T)$, that is $C_{\mathrm{BS}}=C_{\mathrm{BS}}\left(P^{d}(0, T) F_{T}, y(K, T), w(y(K, T), T)\right)$, the first two derivatives of the call price with respect to the strike can be computed as

$$
\begin{aligned}
\frac{\partial C_{\mathrm{BS}}}{\partial K}= & \left(\frac{\partial C_{\mathrm{BS}}}{\partial y}+\frac{\partial C_{\mathrm{BS}}}{\partial w} \frac{\partial w}{\partial y}\right) \frac{\partial y}{\partial K} \\
\frac{\partial^{2} C_{\mathrm{BS}}}{\partial K^{2}}= & {\left[\frac{\partial^{2} C_{\mathrm{BS}}}{\partial y^{2}}+\left(2 \frac{\partial^{2} C_{\mathrm{BS}}}{\partial w \partial y}+\frac{\partial^{2} C_{\mathrm{BS}}}{\partial w^{2}} \frac{\partial w}{\partial y}\right) \frac{\partial w}{\partial y}+\frac{\partial C_{\mathrm{BS}}}{\partial w} \frac{\partial^{2} w}{\partial y^{2}}\right]\left(\frac{\partial y}{\partial K}\right)^{2} } \\
& +\left(\frac{\partial C_{\mathrm{BS}}}{\partial y}+\frac{\partial C_{\mathrm{BS}}}{\partial w} \frac{\partial w}{\partial y}\right) \frac{\partial^{2} y}{\partial K^{2}} .
\end{aligned}
$$

Since $\frac{\partial y}{\partial K}=\frac{1}{K}$ and $\frac{\partial^{2} y}{\partial K^{2}}=-\frac{1}{K^{2}}$ the second expression can be written as

$$
K^{2} \frac{\partial^{2} C_{\mathrm{BS}}}{\partial K^{2}}=\frac{\partial^{2} C_{\mathrm{BS}}}{\partial y^{2}}+\left(2 \frac{\partial^{2} C_{\mathrm{BS}}}{\partial w \partial y}+\frac{\partial^{2} C_{\mathrm{BS}}}{\partial w^{2}} \frac{\partial w}{\partial y}-\frac{\partial C_{\mathrm{BS}}}{\partial w}\right) \frac{\partial w}{\partial y}+\frac{\partial C_{\mathrm{BS}}}{\partial w} \frac{\partial^{2} w}{\partial y^{2}}-\frac{\partial C_{\mathrm{BS}}}{\partial y} .
$$

The right hand side of this equation demands evaluation of the derivatives of the call price with respect to the log-moneyness and the total implied variance. Using the identity 
$N^{\prime}\left(d_{1}\right)=e^{y} N^{\prime}\left(d_{2}\right)$ we compute the first $w$-derivative as

$$
\begin{aligned}
\frac{\partial C_{\mathrm{BS}}}{\partial w} & =P^{d}(0, T) F_{T}\left[N^{\prime}\left(d_{1}\right) \frac{\partial d_{1}}{\partial w}-e^{y} N^{\prime}\left(d_{2}\right) \frac{\partial d_{2}}{\partial w}\right] \\
& =\frac{1}{2} P^{d}(0, T) F_{T} e^{y} N^{\prime}\left(d_{2}\right) w^{-\frac{1}{2}}
\end{aligned}
$$

and, since $N^{\prime \prime}(x)=-x N^{\prime}(x)$, the second $w$-derivative evaluates as

$$
\begin{aligned}
\frac{\partial^{2} C_{\mathrm{BS}}}{\partial w^{2}} & =\frac{1}{2} P^{d}(0, T) F_{T} e^{y}\left[-N^{\prime}\left(d_{2}\right) d_{2} \frac{\partial d_{2}}{\partial w} w^{-\frac{1}{2}}-\frac{1}{2} N^{\prime}\left(d_{2}\right) w^{-\frac{3}{2}}\right] \\
& =\frac{1}{2} \frac{\partial C_{\mathrm{BS}}}{\partial w}\left[-\frac{1}{4}-\frac{1}{w}+\frac{y^{2}}{w^{2}}\right] .
\end{aligned}
$$

Furthermore, the remaining derivatives are

$$
\begin{aligned}
\frac{\partial^{2} C_{\mathrm{BS}}}{\partial w \partial y} & =\frac{1}{2} P^{d}(0, T) F_{T} e^{y} N^{\prime}\left(d_{2}\right) w^{-\frac{1}{2}}\left[-d_{2} \frac{\partial d_{2}}{\partial y}+1\right] \\
& =\frac{\partial C_{\mathrm{BS}}}{\partial w}\left[-\frac{y}{w}+\frac{1}{2}\right], \\
\frac{\partial C_{\mathrm{BS}}}{\partial y} & =P^{d}(0, T) F_{T}\left[N^{\prime}\left(d_{1}\right) \frac{\partial d_{1}}{\partial y}-e^{y} N\left(d_{2}\right)-e^{y} N^{\prime}\left(d_{2}\right) \frac{\partial d_{2}}{\partial y}\right] \\
& =-P^{d}(0, T) F_{T} e^{y} N\left(d_{2}\right), \\
\frac{\partial^{2} C_{\mathrm{BS}}}{\partial y^{2}} & =-P^{d}(0, T) F_{T} e^{y}\left[N\left(d_{2}\right)+N^{\prime}\left(d_{2}\right) \frac{\partial d_{2}}{\partial y}\right] \\
& =\frac{\partial C_{\mathrm{BS}}}{\partial y}+2 \frac{\partial C_{\mathrm{BS}}}{\partial w} .
\end{aligned}
$$

Plugging in equations (A.3), (A.4), (A.5), (A.6), and (A.7) into (A.2) we arrive at

$$
\frac{1}{2} K^{2} \frac{\partial^{2} C_{\mathrm{BS}}}{\partial K^{2}}=\frac{\partial C_{\mathrm{BS}}}{\partial w}\left[1-\frac{y}{w} \frac{\partial w}{\partial y}+\frac{1}{2} \frac{\partial^{2} w}{\partial y^{2}}+\frac{1}{4}\left(\frac{\partial w}{\partial y}\right)^{2}\left(-\frac{1}{4}-\frac{1}{w}+\frac{y^{2}}{w^{2}}\right)\right] .
$$

Finally, we use the identities

$$
\begin{array}{r}
\frac{\partial y}{\partial T}=-\frac{S_{0}}{F_{T}} \frac{\partial \frac{P^{f}(0, T)}{P^{d}(0, T)}}{\partial T}=f^{f}(0, T)-f^{d}(0, T), \\
\frac{\partial\left(P^{d}(0, T) F_{T}\right)}{\partial T}=S_{0} \frac{\partial\left(P^{f}(0, T)\right)}{\partial T}=-f^{f}(0, T) P^{d}(0, T) F_{T}
\end{array}
$$

to formulate the time derivative of the call price as

$$
\frac{\partial C_{\mathrm{BS}}}{\partial T}=-f^{f}(0, T) C_{\mathrm{BS}}+\frac{\partial C_{\mathrm{BS}}}{\partial w} \frac{\partial w}{\partial T}+\left(\frac{\partial C_{\mathrm{BS}}}{\partial y}+\frac{\partial C_{\mathrm{BS}}}{\partial w} \frac{\partial w}{\partial y}\right)\left(f^{f}(0, T)-f^{d}(0, T)\right) .
$$


Plugging in equations (A.8) and (A.9) into (2.18) gives us the extended Dupire formula in the log-moneyness/total implied variance parametrization.

$$
\sigma^{\mathrm{LV}}(K, T)^{2}=\frac{\frac{\partial C_{\mathrm{BS}}}{\partial T}-P^{d}(0, T) \mathbf{E}^{\mathbb{Q}^{\mathrm{T}}}\left[\left(K r_{T}^{d}-S_{T} r_{T}^{f}\right) \mathbb{1}_{S_{T}>K}\right]}{\frac{\partial C_{\mathrm{BS}}}{\partial w}\left[1-\frac{y}{w} \frac{\partial w}{\partial y}+\frac{1}{2} \frac{\partial^{2} w}{\partial y^{2}}+\frac{1}{4}\left(\frac{\partial w}{\partial y}\right)^{2}\left(-\frac{1}{4}-\frac{1}{w}+\frac{y^{2}}{w^{2}}\right)\right]},
$$

where the explicit forms of $C_{\mathrm{BS}}, \frac{\partial C_{\mathrm{BS}}}{\partial w}, \frac{\partial C_{\mathrm{BS}}}{\partial y}$, and $\frac{\partial C_{\mathrm{BS}}}{\partial T}$ are given by (A.1), (A.3), (A.6), and (A.9) respectively.

Equation (3.11) allows us to write the extended Dupire formula for the two stochastic rates and stochastic local volatility model (3.9) in the total implied variance surface formulation as well. Since the deterministic local volatility limiting case was already computed in this formulation as in (A.10), we can write the leverage function for the stochastic local volatility generalization as

$$
L_{s}(K, T)^{2}=\frac{\frac{\partial C_{\mathrm{BS}}}{\partial T}-P^{d}(0, T) \mathbf{E}^{\mathbb{Q}^{\mathrm{T}}}\left[\left(K r_{T}^{d}-S_{T} r_{T}^{f}\right) \mathbb{1}_{S_{T}>K}\right]}{\frac{\partial C_{\mathrm{BS}}}{\partial w}\left[1-\frac{y}{w} \frac{\partial w}{\partial y}+\frac{1}{2} \frac{\partial^{2} w}{\partial y^{2}}+\frac{1}{4}\left(\frac{\partial w}{\partial y}\right)^{2}\left(-\frac{1}{4}-\frac{1}{w}+\frac{y^{2}}{w^{2}}\right)\right] \mathbf{E}^{\mathbb{Q}^{\mathrm{T}}}\left[U_{T} \mid S_{T}=K\right]} .
$$

Single stochastic rate limit In the limit where the foreign rates $r_{T}^{f}$ are deterministic, this equation becomes

$$
\sigma^{\mathrm{LV}}(K, T)^{2}=\frac{\frac{\partial C_{\mathrm{BS}}}{\partial w} \frac{\partial w}{\partial T}-f^{d}(0, T)\left(\frac{\partial C_{\mathrm{BS}}}{\partial y}+\frac{\partial C_{\mathrm{BS}}}{\partial w} \frac{\partial w}{\partial y}\right)-P^{d}(0, T) K \mathbf{E}^{\mathbb{Q}^{\mathrm{T}}}\left[r_{T}^{d} \mathbb{1}_{S_{T}>K}\right]}{\frac{\partial C_{\mathrm{BS}}}{\partial w}\left[1-\frac{y}{w} \frac{\partial w}{\partial y}+\frac{1}{2} \frac{\partial^{2} w}{\partial y^{2}}+\frac{1}{4}\left(\frac{\partial w}{\partial y}\right)^{2}\left(-\frac{1}{4}-\frac{1}{w}+\frac{y^{2}}{w^{2}}\right)\right]} .
$$

Deterministic rates limit In the limit where both the domestic rates $r_{T}^{d}$ and the foreign rates $r_{T}^{f}$ are deterministic, the equation further simplifies to the form given in [20]

$$
\sigma^{\mathrm{LV}}(K, T)^{2}=\frac{\frac{\partial w}{\partial T}}{1-\frac{y}{w} \frac{\partial w}{\partial y}+\frac{1}{2} \frac{\partial^{2} w}{\partial y^{2}}+\frac{1}{4}\left(\frac{\partial w}{\partial y}\right)^{2}\left(-\frac{1}{4}-\frac{1}{w}+\frac{y^{2}}{w^{2}}\right)} .
$$

\section{Acknowledgments}

The authors are grateful to the SIAM SIFIN reviewers and the editor for their valuable comments that have substantially improved the paper. The authors are indebted to Dooheon Lee for numerous enlightening discussions and guidance about the construction and the flow of this paper. The authors would also like to thank Agus Sudjianto for supporting this research, and Vijayan Nair for suggestions, feedback, and discussion regarding this work. Orcan Ogetbil is appreciative for Paul Feehan's original introduction to the subject and his endorsement. Any opinions, findings and conclusions or recommendations expressed in this material are those of the author and do not necessarily reflect the views of Wells Fargo Bank, N.A., its parent company, affiliates and subsidiaries. 


\section{References}

[1] Bruno Dupire. Pricing with a Smile. Risk Magazine, pages 18-20, 11994.

[2] Emanuel Derman and Iraj Kani. Riding on a Smile. Risk Magazine, pages 32-39, 2 1994.

[3] Leif Andersen and Jesper Andreasen. Jump-Diffusion Processes: Volatility Smile Fitting and Numerical Methods for Option Pricing. Review of Derivatives Research, $4(3): 231-262,2000$.

[4] Eric Benhamou, Arnaud Rivoira, and Anne Gruz. Stochastic Interest Rates for Local Volatility Hybrids Models, 2008.

[5] Bing Hu. Local Volatility Model with Stochastic Interest Rate. Master's thesis, York University, 82015.

[6] Michal Jex, R. C. W. Henderson, and Desheng Wang. Pricing Exotics Under the Smile. Risk Magazine, 12:72-75, 111999.

[7] Alexander Lipton. The vol smile problem. Risk Magazine, 15(2):61-66, 22002.

[8] Yu Tian, Zili Zhu, Geoffrey Lee, Fima Klebaner, and Kais Hamza. Calibrating and Pricing with a Stochastic-Local Volatility Model. The Journal of Derivatives, 22(3):2139,2015 .

[9] Ben M. Hambly, Matthieu Mariapragassam, and Christoph Reisinger. A forward equation for barrier options under the Brunick \& Shreve Markovian projection. Quantitative Finance, 16:827 - 838, 2014.

[10] Julien Guyon and Pierre Henry-Labordère. Being particular about calibration. Risk Magazine, 2012.

[11] Griselda Deelstra and Grégory Rayée. Local Volatility Pricing Models for Long-dated FX Derivatives. Applied Mathematical Finance, 20(1204.0633):380-402, 2013.

[12] W. Feller. On the Theory of Stochastic Processes, with Particular Reference to Applications. In Proceedings of the [First] Berkeley Symposium on Mathematical Statistics and Probability, pages 403-432, Berkeley, Calif., 1949. University of California Press.

[13] John C. Cox, Jonathan E. Ingersoll, and Stephen A. Ross. A Theory of the Term Structure of Interest Rates. Econometrica, 53(2):385-407, 1985.

[14] G. Tataru, T. Fisher, and J. Yiu. The Bloomberg Stochastic Local Volatility Model For FX Exotics. Bloomberg, 2012.

[15] István Gyöngy. Mimicking the one-dimensional marginal distributions of processes having an Ito differential. Probability Theory and Related Fields, 71:501-516, 1986.

[16] P. S. Hagan and Diana E. Woodward. Markov interest rate models. Applied Mathematical Finance, 6:233-260, 1999. 
[17] Roland Lichters, Roland Stamm, and Donal Gallagher. Modern derivatives pricing and credit exposure analysis: theory and practice of CSA and XVA pricing, exposure simulation and backtesting. Springer, 2015.

[18] Matthias R. Fengler. Arbitrage-free smoothing of the implied volatility surface. Quantitative Finance, 9(4):417-428, 2009.

[19] Jim Gatheral and Antoine Jacquier. Arbitrage-free SVI volatility surfaces. Quantitative Finance, 14(1):59-71, 2014.

[20] Jim Gatheral. The Volatility Surface, chapter 1, pages 1-14. John Wiley \& Sons, Ltd, 2012 . 